\title{
INTEGRATING MICROFLUIDIC SYSTEMS AND NMR SPECTROSCOPY: PRELIMINARY RESULTS
}

\author{
Jonathan D. Trumbull, Ian K. Glasgow, David J. Beebe, and Richard L. Magin \\ Department of Electrical and Computer Engineering \\ Beckman Institute for Advanced Science and Technology \\ University of Illinois at Urbana-Champaign \\ 405 North Mathews Avenue \\ Urbana, IL 61801
}

\begin{abstract}
The philosophy of miniature total analysis systems ( $\mu$-TAS) hinges on the integration of multiple chemical processing steps and the means of analyzing their results on the same miniaturized system. We have investigated coupling chip-based capillary electrophoresis (CE) with nuclear magnetic resonance spectroscopy (NMR) bringing unprecedented structural analysis capability to $\mu$-TAS systems. In this paper, we present our initial experimental results and conclusions which are applicable to a wide range of microfluidic systems. Linewidths of $1.4 \mathrm{~Hz}$ have been demonstrated using single turn planar NMR coils integrated with microfluidic channels.
\end{abstract}

\section{INTRODUCTION}

The use of chip-based capillary electrophoresis systems offer substantial advantages over more conventional implementations only if they allow either parallel operations to be performed, provide a needed size advantage, or integrate with subsequent processes. This paper is concerned with the integration of $\mathrm{CE}$ chips with NMR and the associated benefits and difficulties. A brief description of pulse NMR [1] will be given followed by details specific to volume limited samples. After this background information, we will describe the fabrication and testing of two fluidic-NMR devices. No description of the electrokinetic effects, electroosmotic flow (EOF) and electrophoresis, encountered in CE systems will be given since excellent references are available for both general information $[2,3]$ and details specific to on-chip CE [4-6].

\section{BACKGROUND}

NMR, although often described classically, is a quantum phenomenon. When a nucleus possessing a magnetic moment is placed in a static magnetic field, it exists in quantized orientations. For the ${ }^{1} \mathrm{H}$ nucleus, the alignment can be parallel or antiparallel to the static field with the antiparallel case having slightly higher energy. Because the energy difference is small compared to the average thermal energy of the system, a Boltzman distribution results with only a slight excess of protons being in the lower energy state $\left(\sim 10^{-5}\right.$ of the entire sample at typical field strengths). Protons may be excited into the high energy state by the application of a short pulse from a RF coil. As the system decays to its equilibrium state, it produces an exponentially damped signal called the free induction decay (FID). The contribution to the FID from each part of the sample volume has a frequency that is linearly dependent upon the value of the static magnetic field at that location. Because the local field is influenced by the chemical environment the proton is in, the FID will be composed of different frequencies corresponding to the various functional groups on the compound. In 1-D NMR, this information is represented by its frequency spectrum with the contributions of different functional groups showing up as "lines". More sophisticated pulse sequences and analysis (2-D NMR) provide the researcher with the means to determine the structure of the molecule in question.

For NMR to become a useful tool for the analysis of volume limited samples, several obstacles must be overcome. First since the excess population of low energy protons is so small, the signal is very weak. Thus it is necessary to couple the sample strongly to the coil by filling as much of the coil's interrogation volume as possible (fill factor). Significant progress has been made towards understanding the scaling of coupling for microcoils $[7,8]$ and is summarized by Webb [9].

A conflicting constraint to maximizing the fill factor is the requirement that the coil not affect the homogeneity of the static field since the lines in the NMR spectrum would be broadened and the resolution decreased. To meet this requirement, the coil must be positioned away from the sample (which decreases the sensitivity) or made from a material whose magnetic properties match that of the surroundings. This can be done with relative ease by layering metals to provide a net zero magnetic susceptibility $[10,11]$. The more obvious solution, creating an alloy of these materials, cannot be used because of the large decrease in conductivity in otherwise suitable metal combinations [12]. Another approach is to match the surroundings to the coil through a susceptibility matching fluid [7].

NMR has been combined successfully with high performance liquid chromatography (HPLC) [13-16] but faces serious problems as a detector in CE applications because of the lower sample volumes required to prevent Joule heating [2]. One of the first reported discrete CE-NMR systems required injection concentrations approaching $1 \mathrm{M}$ for reasonable online detection times [17]. Given that the detection limits for laser induced fluorescence (LIF) extend to the $10^{-12} \mathrm{M}$ range [18], this is clearly unacceptable. If the role of NMR were changed to solely that of an analyzer of accumulated fractions, much longer acquisition times would be tolerable and lower concentrations therefore permissible. Automated CE fraction collection has been previously accomplished using capillary tubes and external components [19], but a more elegant method is that of a microfabricated collection system [20]. Such a system would allow the separations to be performed using a conventional detection scheme (i.e. UV absorbance or fluorescence) while collecting selected fractions into sample reservoirs with integral NMR receiver coils. After a sufficient amount of the pure substances were obtained, the entire system could be placed into a superconducting magnet and the compound's spectra obtained.

\section{FABRICATION}

The first system, "Trident", was designed to be a proof of concept fluidic-NMR device to gauge the effectiveness of integrated, single-turn planar NMR coils. Although less sensitive than solenoidal coils, from a fabrication perspective they are easier to produce than solenoidal or saddle coils.

Referring to Figure 1, the channels consist of polyimide layers between a $50 \times 75 \mathrm{~mm}$ glass microscope slide and a $45 \times 70$ $\mathrm{mm} \times$ no. 1 thickness $(0.15 \mathrm{~mm})$ cover glass. The polyimide types used, Pyralin@ PD PI-2721 and PI-2723 (Du Pont Electronics, Wilmington, DE), are negative tone photodefineable polyimide precursors. First, $2 \mathrm{~mm}$ holes were drilled through the slide, and the channel bottom was formed by spin coating a layer of PI-2723. The roughly $30 \mu \mathrm{m}$ thick walls of the channels consisted of two 
pattemed PI-2721 layers. Next, PI-2723 was spin coated onto the cover glass and soft baked. To solvent bond this "top" layer to the patterned "walls" layer on the slide, a mixture of 4 parts by volume of Pyralin solvent T-9039 to 1 part PI-2723 was spin coated at $7000 \mathrm{rpm}$ for 30 seconds onto the top layer. Immediately, this cover glass was pressed firmly onto the slide so that the solvent layer was now between the top and wall layers. Finally, the polyimide was cured in a tube furnace (Lindberg/Blue $M$, Asheville, NC).

A lift-off process was used to pattern the coil on the outer surface of the cover glass. The metal was formed from 3 evaporated layers: $\mathrm{Cr} / \mathrm{Cu} / \mathrm{Cr}$ with respective thicknesses of 150 , 9700 , and $150 \AA$. The resistance of the coil, pad to pad, was measured to be 5.9 ohms. Acrylic wells were then placed over the drilled holes and bonded with epoxy. The completed system is shown in Figure 2.

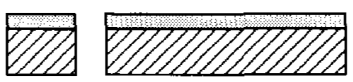

Apply Polyimide on Glass Slide
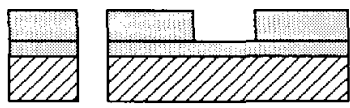

Pattern 2nd Layer of Polyimide

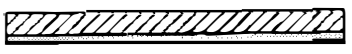

Prepare a Glass Cover with

Polyimide and Solvent

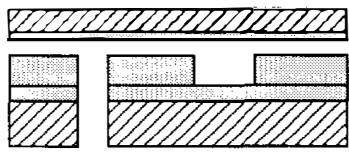

Press Together and Cure in a Furnace

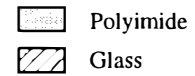

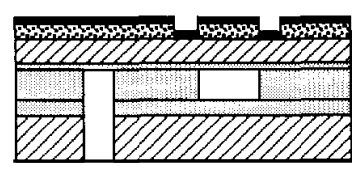

Add Photoresist and Metal Layers

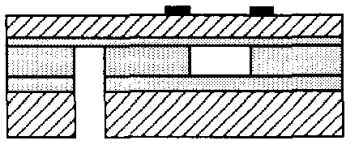

Remove Photoresist

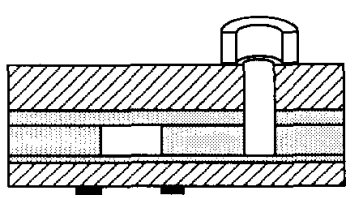

Adhere Wells

Coil $(\mathrm{Cr} / \mathrm{Cu} / \mathrm{Cr})$

Photoresist
Figure 1: Fabrication steps used to produce the polyimide device ("Trident").

The second device type created (Figure 3), "SpinCollector" was made from etched glass capillaries using methods developed from $[4,5] .1 \mathrm{~mm}$ thick, annealed Pyrex glass wafers were etched in $\mathrm{HF}$ and $\mathrm{HNO}_{3}$ to a depth of $20 \mu \mathrm{m}$ through a $\mathrm{Cr} / \mathrm{Au}$ mask. Access holes were drilled ultrasonically and the mask was stripped. They were then cleaned in a $1 \%$ HF bath for 1 minute with ultrasonic agitation to remove any loose glass particles [21]. After thorough cleaning [5], the wafers were thermally bonded to unprocessed pieces forming closed channels. Initially $150 \mu \mathrm{m}$ thick Pyrex was used as a cover piece, but repeated bonding problems due to a slight bending of the thin glass forced us to use standard $1 \mathrm{~mm}$ pieces. This bonding problem has since been solved by using a sodium silicate low temperature process [22] which pulls the two surfaces together quite nicely.

A coil was then formed through a lift-off process on the undrilled side over the disk-shaped reservoir, and glass wells were attached using epoxy.

Both "Trident" and "SpinCollector" designs were fitted to printed circuit board (PCB) bases containing tuning capacitors. Connections between the PCB and the coil were made with 24 AWG magnet wire and conductive epoxy (Epo-tek H20E, Billerica, MA).

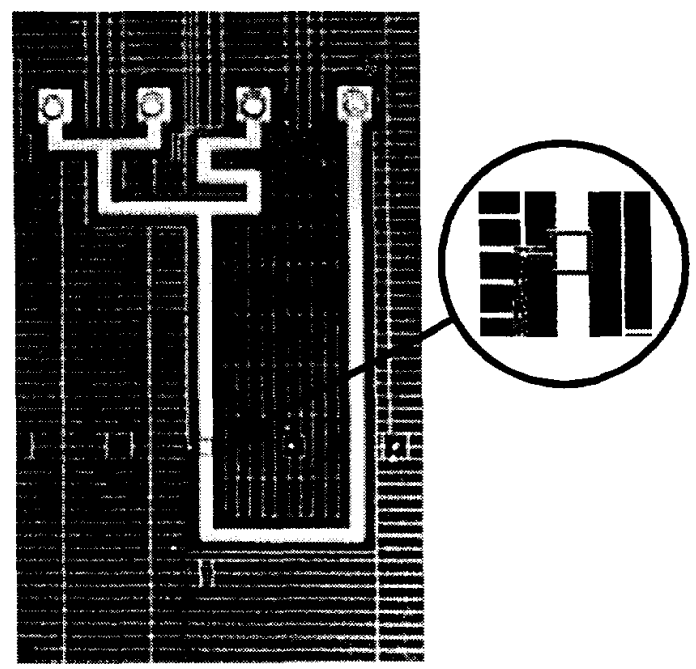

Figure 2: Completed "Trident" polyimide structure with blowup of the NMR receiver coil which is $1 \mathrm{~mm}$ on a side (inner) with a trace width of $200 \mu \mathrm{m}$. Assuming a channel depth of $30 \mu \mathrm{m}$, the coil encloses a sample volume of $30 \mathrm{~nL}$. Note that the fluidic channels are the thicker lines connected to the access holes on the top. The other lines are for solvent venting during curing.

(b)

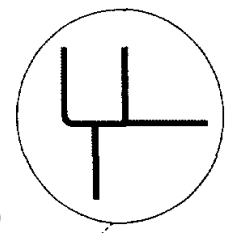

(a)
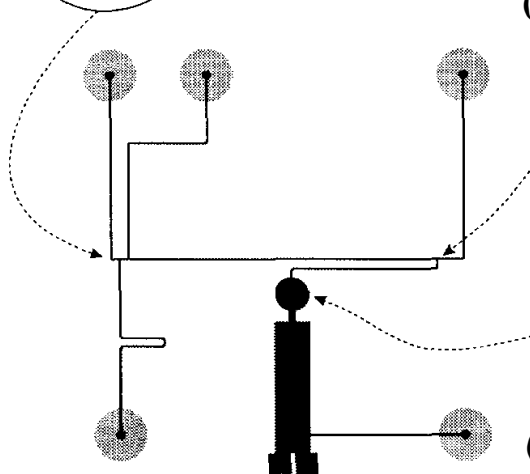

(d)

(c)

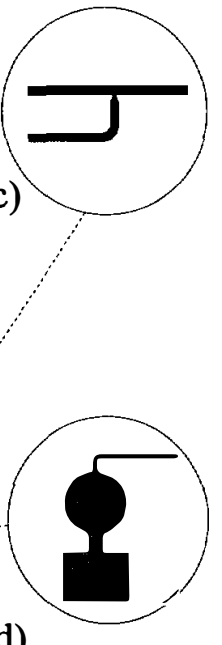

Figure 3: Mask layout for "SpinCollector". (a) The complete device. The 5 wells are located on the periphery interconnected by the $200 \mu \mathrm{m}$ wide, $20 \mu \mathrm{m}$ deep channels in glass. Close up views of: (b) the "double- $T$ " injector, (c) collection junction, and $(d)$ the collection reservoir with integrated NMR coil.

\section{METHODS}

To quantify the SNR and linewidth of both the "Trident" and "SpinCollector" designs, each was loaded with deionized water, matched to $50 \Omega$ using a network analyzer, and inserted into the bore of a 5.9 Tesla $(250 \mathrm{MHz})$ Oxford/89-mm superconducting magnet (Oxford Instruments, UK). The integrated planar coil was used for both the $90^{\circ}$ transmit pulse and the received FID (bandlimited to $1000 \mathrm{~Hz}$ ). 4096 time points were sampled and Fourier transformed. Signal to noise was determined by measuring the peak height and comparing it to the RMS noise. Linewidth was found by fitting the peak to a Lorentzian curve and finding the width at half height. 
Fraction collection in the "SpinCollector" device was tested using two amino acids, arginine and alanine, at a pH of 9.0 where the difference in charge facilitates the separation. Tagging was accomplished using a standard fluorescein isothiocyanate (FITC; Molecular Probes, Eugene, OR) protocol [18]. $3.0 \mathrm{mM}$ concentrations of the amino acids in $50 \mathrm{mM}$ borate buffer were added to an equal amount of $3 \mathrm{mM}$ FITC dissolved in acetone $(0.1 \%$ pyridine $)$. After reacting overnight in a light-tight box, the acetone was pulled off under vacuum and the borate run buffer was degassed using an ultrasonic bath. Both the run buffer and the sample were filtered to $0.2 \mu \mathrm{m}$ (Millipore, Bedford, MA) and introduced to the wells.

The experimental setup used is depicted in Figure 4. High voltage power supplies (MJ15N100; Glassman, Whitehouse Station, NJ) switched by Kilovac (Santa Barbara, CA) K43C234 $10 \mathrm{kV}$ relays provided the potential needed to drive the injection, separation, and fraction collection. The control software was written in Visual Basic 5.0 (Microsoft, Redmond, WA) using ComponentWorks 1.1 and an AT-AO-6 control board both from National Instruments (Austin, TX). Visualization was provided by an Olympus BX60 epi-fluorescent microscope and a PentaMAX intensified CCD camera (Princeton Instruments, Trenton, NJ). Selected pictures taken with the camera are presented in Figure 6. No NMR spectra were taken of collected samples due to the low concentrations necessary for tagging (FITC precipitates in aqueous solutions at required concentrations).

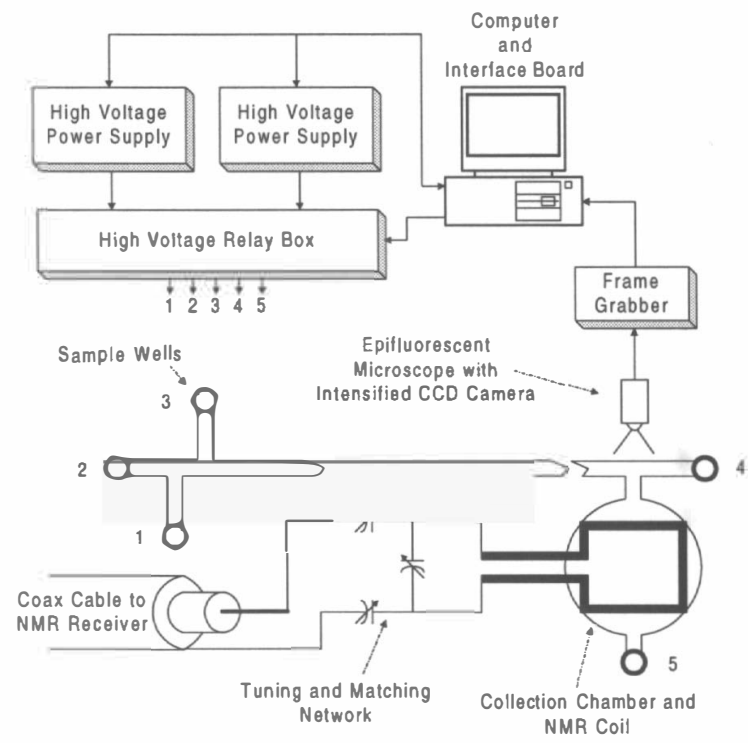

Figure 4: Setup for separation and fraction collection.

The conceptual sequence of events needed to collect one fraction is detailed in Figure 5 and is similar to that used in [20]. A $1 \mathrm{~mm}$ long $(\sim 2 \mathrm{~nL})$ plug is defined in (a) by applying a voltage across the top and bottom left wells causing a net motion upward. By switching the voltage, the individual components move to the right (shown in (b)) at a velocity characteristic of their charge/mass ratios. After becoming distinguishable bands in (c), the front analyte is detoured to the reservoir in (d). The separation column is cleared in (e) to make way for the next run. A slight efflux is maintained while the second band passes by to prevent contamination of the collected sample. By running many such cycles, enough of the "unknown" could be accumulated for NMR analysis. (a)

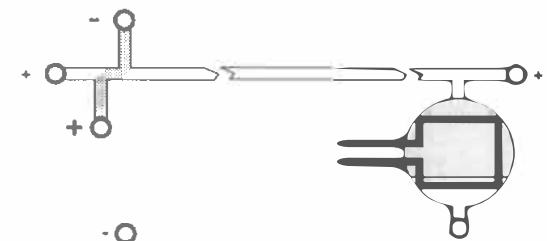

(b)

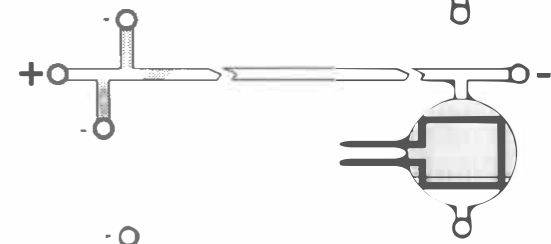

(c)

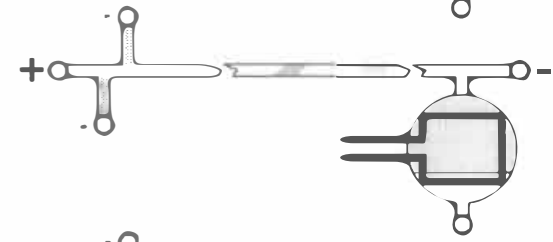

(d)

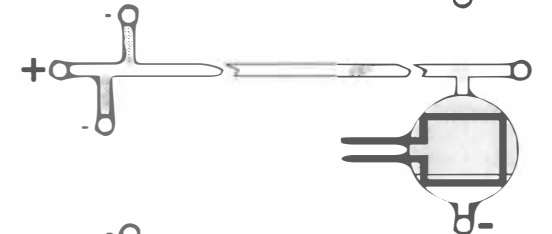

(e)

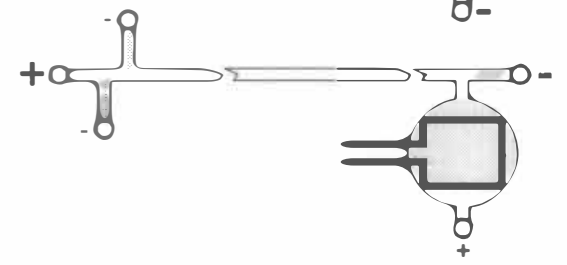

Figure 5: Schematic representation of (a) plug injection, (b) initial separation, (c) further separation, (d) collection of desired analyte, (e) clearing column. Note that the relative size of the "+" and "-" characters indicates the magnitude of the potential.

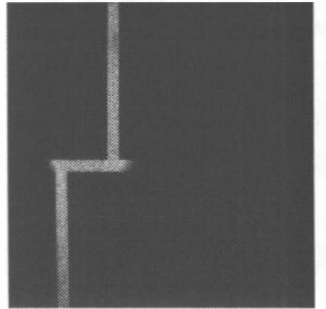

(a)

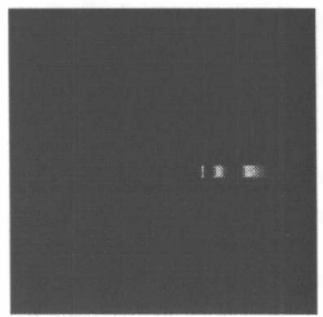

(c)

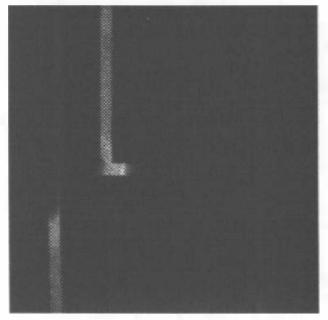

(b)

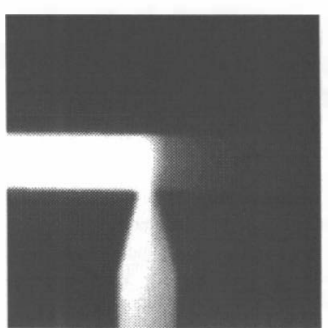

(d)
Figure 6: A selected iteration of the separation and collection sequence imaged with an intensified $C C D$ and epi-fluorescent microscope: (a) plug is defined, (b) separation starts, (c) distinct bands formed (ARG, ALA, and unbound FITC), (d) selected plug is collected. This is repeated until substantial sample accumulates in the reservoir: 


\section{RESULTS}

The SNR for the "Trident" polyimide system, shown in Figure $7(a)$, was $23.5 /$ scan with a linewidth of $1.4 \mathrm{~Hz}$ that is the narrowest for any microfabricated planar coil to date including those using a susceptibility matching fluid [23]. It was apparent, however, that the SNR was significantly lower than required for the system to be used as an online detector of reasonable concentrations (water is $55 \mathrm{M}$ ).

Although the "SpinCollector" design held $393 \mathrm{~nL}$ of sample, the coil was significantly farther away $(1 \mathrm{~mm}$ versus $150 \mu \mathrm{m})$ which resulted in only a slight increase in SNR to $41 /$ scan with a linewidth of $3.1 \mathrm{~Hz}$.

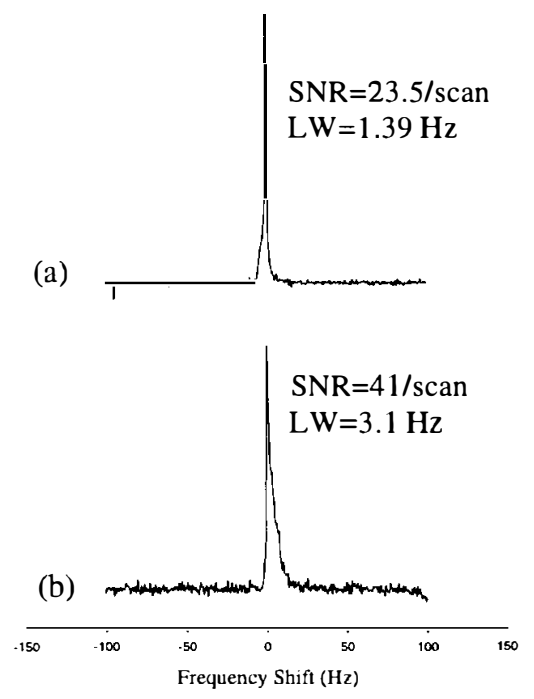

Figure 7: NMR spectra of $\mathrm{H}_{2} \mathrm{O}$ for (a) "Trident" with 16 scans signal averaged giving a 23.5 SNR per scan and a linewidth of $1.39 \mathrm{~Hz}$, (b) "SpinCollector" with a linewidth of $3.1 \mathrm{~Hz}$ and SNR of 41 for a single scan

\section{DISCUSSION}

Trying to detect analytes would still prove difficult at these SNR. For example, identifying $100 \mathrm{mM}$ arginine using "SpinCollector" would take approximately 6 hours. Note that the acquisition time increases as the square of the concentration decreases so the time required for the same volume of $100 \mu \mathrm{M}$ amino acid would take almost 700 years (we will post the exact results at Hilton Head 2682 barring unforeseen circumstances)! Also, keep in mind that this does compare favorably with conventional 5 and $10 \mathrm{~mm}$ NMR saddle coils in terms of mass sensitivity [7].

The low SNR of the two tested devices is a direct result of their geometry. A square planar coil has a sensitive region that extends out over half its effective radius. While allowing a larger volume to be interrogated, it also means that our choice of a thin channel above the coil amounts to a sub-optimal filling factor. There are two possible solutions. First, change the sample volume from a slice to a dome shaped region fully contained within the "sweet-spot" of the coil. This would increase the total signal but with the added expense of needing additional sample. A more sophisticated method involves "pulling" the sensitive region in closer to the coil so that the SNR for a given unit volume increases. The simplest way of shaping the field with a rectangular geometry is to reduce the smallest dimension as shown in Figure 8.

For a thin sample directly above a $5 \mathrm{~mm}$ coil, the sensitivity per unit volume increases by a factor of 65 as the coil shrinks to $100 \mu \mathrm{m}$. What is needed is a surface coil configuration that approximates the field pattern of an array of these smaller coils without dramatically increasing the resistance. Given this 65 fold increase in sensitivity, the acquisition time would drop $65^{2}=4225$ times!

Even having this hypothetical performance increase, the planar element cannot be compared to solenoidal coils in the same sample size regime $[7,9]$ whose limits of detection stretch to the low picomole arena given mere minutes of acquisition time. Planar coils do, however, provide a significant improvement over standard NMR mass detection limits for small volumes and are easily integrated into batch fabricated analytical devices. Discrete wound solenoids provide a drastic improvement in sensitivity, but are difficult to integrate with planar processes.

Regardless of the choice of planar versus solenoid, the ability to perform additional steps or mechanics are important for some applications.. For example, combinatorial chemistry is typically accomplished on small resin beads that serve as the solid support for tethered compounds present in quantities of 100's of picomoles. For a high resolution spectra to be obtained, the compounds must be cleaved from the beads, or the sample must be spun in the coil [24]. Cleaving is a "destructive" process and spinning at $\mathrm{kHz}$ range frequencies on a chip would prove challenging at best. If high concentrations of liquid phase analytes can be used (or if enrichment can be performed on chip [25]), planar NMR coils offer more than just easy integration with other processes. By shrinking the sample to the nanoliter range, the volume over which the magnetic field must be uniform decreases appropriately allowing either a smaller (much less expensive) magnet to be used, or multiple spectrums to be taken in parallel in a conventional magnet (multiplying throughput). With the large initial expenditure for a high-field superconducting magnet and the associated cost of ownership, these benefits are significant with volume limited samples.

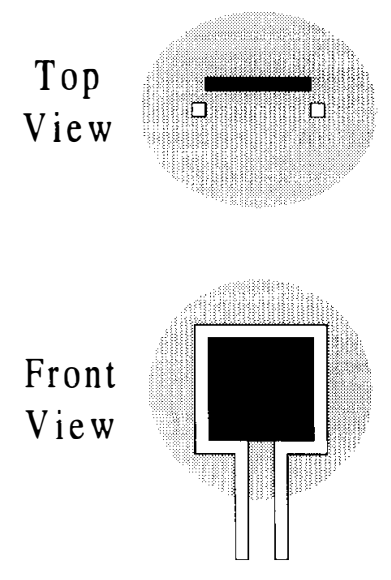

(a)

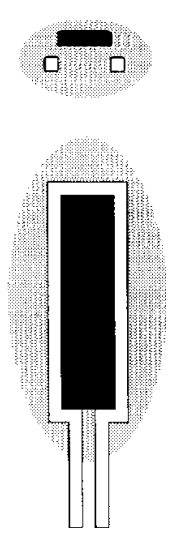

(b)
Figure 8: Increasing the sensitivity by altering the geometry of sample (black) and coil (white) to shrink the interrogation region (gray): (a) square planar coil, (b) rectangular planar coil with equivalent sample volume.

\section{CONCLUSIONS}

It has been shown that the integration of NMR into $\mu$-TAS is feasible from a fabrication standpoint. By extrapolating the current experimental results through simulation, improvements are planned that may allow small volumes $(\sim 100 \mathrm{~nL})$ of high $(\sim 10$ $\mathrm{mM}$ ) concentration solutions to be analyzed in reasonable amounts of time ( hours). If the fabrication process can tolerate "pick and place" assembly, lower concentration spectra can be obtained using 
hand wound or nontraditional solenoids [26-28].

\section{ACKNOWLEDGEMENTS}

We would like to thank Timothy L. Peck of the Magnetic Resonance Microsensors company for his valuable help with simulations, Andrew G. Webb, Jeffery E. Stocker, and Juan G. Santiago for their insightful suggestions, and Bruce Flachsbart for the use of "his" fabrication facilities.

\section{REFERENCES}

[1] A. E. Derome, Modern NMR Techniques for Chemistry Research, vol. 6. Oxford: Pergamon Press, (1987), pp. 1-96.

[2] J. Jorgenson and K. D. Lukacs, "Zone Electrophoresis in Open-Tubular Glass Capillaries", Analytical Chemistry, 53, 1298-1302, (1981)

[3] G. M. Janini, and Haleem J. Issaq, "The Buffer in Capillary Zone Electrophoresis," in Capillary Electrophoresis, N. A. Guzman, New York: Marcel Dekker, Inc., 1993, pp. 119. 160.

[4] D. J. Harrison, A. Manz, Z. Fan, H. Ludi, and H. M. Widmer, "Capillary Electrophoresis and Sample Injection Systems Integrated on a Planar Glass Chip", Analytical Chemistry, 64, 1926-1932, (1992).

[5] N. Chiem and D. J. Harrison, "Microchip-Based Capillary Electrophoresis for Immunoassays: Analysis of Monoclonal Antibodies and Theophylline", Analytical Chemistry, 69, 373-378, (1997).

[6] S. C. Jacobson, R. Hergenröder, L. B. Koutny, R. J. Warmack, and M. J. Ramsey, "Effect of Injection Schemes and Column Geometry on the Performance of Microchip Electrophoresis Devices", Analytical Chemistry, 66, 1107113, (1994).

[7] D. L. Olson, T. L. Peck, A. G. Webb, R. L. Magin, and J. V. Sweedler, "High-Resolution Microcoil ${ }^{1} \mathrm{H}-\mathrm{NMR}$ for MassLimited, Nanoliter-Volume Samples”, Science, 270, 19671970, (1995).

[8] T. L. Peck, R. L. Magin, and P. C. Lauterbur, "Design and Analysis of Microcoils for NMR Microscopy", Journal of Magnetic Resonance, 108, 114-124, (1995).

[9] A. G. Webb, "Radiofrequency Microcoils in Magnetic Resonance", Progress in Nuclear Magnetic Resonance Spectroscopy, 31, 1-42, (1997).

[10] F. O. Zelaya, S. Crozier, S. Dodd, R. McKenna, and D. M. Doddrell, "Measurement and Compensation of Field Inhomogeneities Caused by Differences in Magnetic Susceptibility", Journal of Magnetic Resonance, 115, 131136, (1995).

[11] J. F. Schenck, "The Role of Magnetic Susceptibility in Magnetic Resonance Imaging: MRI Magnetic Compatibility of the First and Second Kinds", Medical Physics, 23, 815850, (1996).

[12] M. B. Bever, "Encyclopedia of Material Science and Engineering", Cambridge: The MIT Press, (1986), pp. 848850 .

[13] G. Schlotterbeck, L. H. Tseng, H. Händel, U. Braumann, and K. Albert, "Direct On-Line Coupling of Capillary HPLC with ${ }^{1} \mathrm{H}$ NMR Spectroscopy in the Nanoliter Scale", Analytical Chemistry, 69, 1421-1425, (1997).

[14] S. A. Korhammer and A. Bernreuther, "Hyphenation of High-Performance Liquid Chromatography (HPLC) and other Chromatographic Techniques (SFC, GPC, GC, CE) with Nuclear Magnetic Resonance: A Review", Fresenius Journal of Analytical Chemistry, 354, 131-135, (1996).
[15] B. Behnke, G. Schlotterbeck, U. Tallarek, S. Strohschein, L. H. Tseng, T. Keller, K. Albert, and E. Bayer, "Capillary HPLC-NMR Coupling: High-Resolution ${ }^{1} \mathrm{H}$ NMR Spectroscopy in the Nanoliter Scale", Analytical Chemistry, 68, 1110-1115, (1996).

[16] N. Wu, A. Webb, T. Peck, and J. Sweedler, "On-Line NMR Detection of Amino Acids and Peptides in Microbore LC", Analytical Chemistry, 67, 3101-3107, (1995).

[17] N. Wu, T. L. Peck, A. G. Webb, R. L. Magin, and J. V. Sweedler, "Nanoliter Volume Sample Cells for ${ }^{1} \mathrm{H}$ NMR: Application to On-Line Detection in Capillary Electrophoresis", Journal of American Chemical Society, 116, 7929-7930, (1994).

[18] J. V. Sweedler, J. B.7 Shear, H. A. Fishman, and R. N. Zare, "Fluorescence Detection in Capillary Zone Electrophoresis Using a Charge-Coupled Device with Time-Delayed Integration", Analytical Chemistry, 63, 496-502, (1991).

[19] D. J. Rose and J. Jorgenson, "Fraction Collector for Capillary Zone Electrophoresis", Journal of Chromatography, 438, 23-34, (1988).

[20] C. S. Effenhauser, A. Manz, and H. M. Widmer, "Manipulation of Sample Fractions on a Capillary Electrophoresis Chip", Analytical Chemistry, 67, 2284-2287, (1995).

[21] O. Diepold, "Smoothing of Ultrasonically Drilled Holes in Borosilicate Glass by Wet Chemical Etching", Journal of Micromechanics and Microengineering : Structures, Devices, and Systems, 6, 29-32, (1996).

[22] B. Quenzer, "Low-Temperature Silicon Wafer Bonding", Sensors and Actuators A, 32, 340-344, (1992).

[23] J. E. Stocker, T. L. Peck, A. G. Webb, M. Feng, and R. L. Magin, "Nanoliter Volume, High-Resolution NMR Microspectroscopy Using a 60- $\mu \mathrm{m}$ Planar Microcoil", IEEE Transactions on Biomedical Engineering, 44, 1122-1127, (1997).

[24] P. A. Keifer, L. Baltusis, D. M. Rice, A. A. Tymiak, and J. N. Shoolery, "A Comparison of NMR Spectra Obtained for Solid-Phase-Synthesis Resins Using Conventional HighResolution, Magic-Angle-Spinning, and High-Resolution Magic-Angle-Spinning Probes", Journal of Magnetic Resonance, Series A, 119, 65-75, (1996).

[25] R. Zhang and S. Hjertén, "A Micromethod for Concentration and Desalting Utilizing a Hollow Fiber, with Special Reference to Capillary Electrophoresis", Analytical Chemistry, 69, 1585-1592, (1997).

[26] J. A. Rogers, R. J. Jackman, and G. M. Whitesides, "Constructing Single- and Multiple-Helical Microcoils and Characterizing Their Performance as Components of Microinductors and Microelectromagnets", IEEE Journal of Microelectromechanical Systems, 6, 184-191, (1997).

[27] J. A. Rogers, R. J. Jackman, G. M. Whitesides, D. L. Olson, and J. V. Sweedler, "Using Microcontact Printing to Fabricate Microcoils on Capillaries for High Resolution Proton Nuclear Magnetic Resonance on Nanoliter Volumes", Applied Physics Letters, 70, 2464-2466, (1997).

[28] A. D. Feinerman, R. E. Lajos, V. White, and D. D. Denton, "X-Ray Lathe: An X-Ray Lithographic Exposure Tool for Nonplanar Objects", Journal of Microelectromechanical Systems, 5, 250-255, (1996). 